\title{
Gender Difference on Stress Induced by Malaria Parasite Infection and Effect of Anti-malaria Drug on Stress Index
}

\author{
ESAN A. $\mathrm{J}^{1, *}$, OMISAKIN C.T ${ }^{1}$, TITILAYO O. E ${ }^{2}$, FASAKIN K. A $^{1}$ \\ ${ }^{1}$ Department of Haematology and Blood Transfusion Science, Federal Medical Centre, Ido-Ekiti Ekiti State \\ ${ }^{2}$ Croydon Grace Diagnostic Cenre, Igando, Lagos State \\ *Corresponding author: ayodelejacob4u@gmail.com
}

Received April 04, 2014; Revised June 05, 2014; Accepted June 05, 2014

\begin{abstract}
Malaria is a serious public health problem in most countries of the tropics. Oxidative stress is related to the severity of malaria, oxidative stress in malaria may originate from several sources including intracellular parasitized erythrocytes and extra-erythrocytes as a result of haemolysis and host response. The aim of this study therefore is to determine the gender difference on stress induced by malaria parasite infection and effect of antimalaria drug on stress index. 202 confirmed malaria infected patients were recruited for the study between the ages of $15-64$ years of both sexes at the general outpatient clinic of the Federal Medical Centre, Ido-Ekiti, Nigeria. 129(63.9\%) were males and 73(36.1\%) were females. The mean \pm SD of MDA, MPC and WBC in male were significantly $(\mathrm{P}<0.05)$ higher compared to female in pre, post anti-malaria drug treatment. Stress induced by malaria parasite was observed higher in male compared to female; gender norms and values that influence the division of labour, leisure patterns, and sleeping arrangements can influence different patterns of exposure to mosquitoes for men and women which responsible for differences in stress induced by malaria parasite among the gender; during malaria treatment, the level of stress induced by malaria parasite was decline due to the effect of antimalaria drug used.
\end{abstract}

Keywords: gender, malaria parasite, stress

Cite This Article: eSAN A. J, OMISAKIN C.T, TITILAYO O. E, and FASAKIN K. A, "Gender Difference on Stress Induced by Malaria Parasite Infection and Effect of Anti-malaria Drug on Stress Index." American Journal of Biomedical Research, vol. 2, no. 3 (2014): 42-46. doi: 10.12691/ajbr-2-3-1.

\section{Introduction}

Malaria is a serious public health problem in most countries of the tropics. It is a major cause of mortality and morbidity, between 300 and 500 million people suffer acute cases of malaria in 100 developing countries each year, and the majority of the victims are children [1]. In Nigeria about 96 million people are exposed to malaria, and out of these 64 million people get infected and almost 300,000 deaths are being reported annually in the general population, of which over 100,000 deaths are of children [2]. However, the available data indicates that malaria is the most common cause of outpatient visits. Malaria is caused by protozoan parasites belonging to the genus plasmodium which infects and destroys red blood cells. Four species of plasmodia (Plasmodium falciparum, Plasmodium malariae, Plasmodium ovale and Plasmodium vivax) cause malaria in humans of which Plasmodium falciparum is the most dangerous form of malaria and life threatening in an unprotected, non-immune population causing high morbidity and mortality. A gender approach contributes to both understanding and combating malaria. Gender norms and values that influence the division of labour, leisure patterns, and sleeping arrangements may lead to different patterns of exposure to mosquitoes for men and women [3]. Detection of malaria in peripheral smear is the gold standard for diagnosis of malaria. Malaria parasite counts is the most important aspects of reporting malaria infections, it estimate the level of parasitemia present in the blood, morphological assessment of malaria parasites is also critical for accurate interpretation. Assessment of the parasite density provides a useful indicator of severity of infection, particularly in non-immune patients, and the level of parasitaemia (parasite burden or load) correlates generally with clinical features and prognosis. There are two acceptable methods of expressing the malaria parasite load: either as the percentage of infected erythrocytes as counted on a stained thin blood film (e.g. 1\% parasitaemia), or the number of parasites per unit volume of blood (e.g.5000 parasites $/ \mu \mathrm{l})$. The latter is usually assessed on a stained thick film by counting parasites against leukocytes $(100,200$ or more), then multiplying by either the patient's own leukocyte count if available, or a standard count of $8000 / \mu 1$ [4]. Stress is a normal physical response to events that makes body feel threatened. Stress response is the way of protecting the body by nervous system responds releasing a flood of stress hormones; including adrenaline and cortisol. These hormones arouse the body for 
emergency action. Stress induce rise in corticotropin releasing hormone $(\mathrm{CRH})$ and adrenocoticotropic hormone (ACTH) which in turn promotes the release of cortisol. The existence of oxidative stress during acute malaria infection has been demonstrated previously, including depletion of antioxidants [5], increased plasma lipid peroxidation and altered fluidity of erythrocyte membrane [6]. The oxidative stress results from host immune reaction, as an acute phase response, and the intraerythrocytic parasite's metabolic processes. Although the oxidative stress appears to be a common phenomenon in acute infection, it may cause a specific consequence in malaria pathogenesis. Oxidative stress is related to the severity of malaria infection and may originate from several sources including intracellular parasitized erythrocytes [7] and extra-erythrocytes as a result of haemolysis and host response [5]. Malondialdehyde is an organic compound being generated from reactive oxygen species (ROS) and as such is assayed in vivo as a biomarker of oxidative stress. Malaria infection has been found to be associated with lipid peroxidation (Malondialdehyde) (MDA) accompanying reduction in antioxidant capacity of the infected patients especially Plasmodium falciparum infection. Artemisinin is widely used as an agent to treat malaria; during recent years this compound and several derivatives have emerged as the most potent anti-malaria agents, being especially active against chloroquine-resistant malaria strains [8]. The mode of action of artemisinin on plasmodium falciparum has been associated with the generation of oxidative stress. During malaria infection, massively recruited by activated monocytes and neutrophils in the host and haemoglobin degradation produced increasing level of reactive oxygen species (oxidative stress). In malaria infection, both host and parasites are under oxidative stress; the effect of reactive oxygen species in malaria can be both beneficial and pathological, depending on the amount and place of production [9] oxidative stress during malaria is beneficial to the patient in the combat against its intra erythrocytic parasites. Several studies have been described in which induction of oxidative stress by treatment with prooxidants proved to be effective against infections. On the other hand, reactive oxygen species play a role in the pathology of malaria, excessive oxidative stress and particularly at unwanted places (for example vascular endothelial lining, blood brain barrier) will damage the defense system. Intra and extra cellular anti-oxidant systems are present to prevent damage but they may fail during disease. Pro-oxidant support the host defense against the parasites when working in or near the infected cells but potentially caused vascular damage when working in or near the vascular lining. Treatment with anti-oxidants rein-forces these systems and protect the patient, especially during the life threatening phase of the disease [10,11]. An understanding of the role and mechanisms of action of oxidative stress may eventually leads to the development of new drugs and new concept of treatment in malaria infection. Oxidative stress is the result of a disturbance in the balance of the naturally generated oxidant and anti-oxidants. This can caused an increase in production of reactive oxygen species and/or a decrease in the activity of anti-oxidant system. Several studies reported on the increased oxidative stress in isolated infected red blood cells (IRBCs). It was suggested that reactive oxygen species are produced as a result of the oxidation and the degradation of ingested haemoglobin in the acidic environment of the parasite's food vacuole. Acidification of the lysate in P.falciparum-infected erythrocytes which also occurs during the digestion of host cell cytosol in the acid food vacuole of the parasite results in the generation of $\left(\mathrm{H}_{2} \mathrm{O}_{2}\right)$. This is probably produced during the auto-oxidation of oxygen haemoglobin. Intact trophozoites infected red blood cells produce hydrogen peroxides $\left(\mathrm{H}_{2} \mathrm{O}_{2}\right)$ and hydrogen hydroxides $(\mathrm{OH})$ radicals about twice as much as normal erythrocytes while no increase is detected at the ring stage when haemoglobin breakdown is limited. In the presence of reducing agents like ascorbate, extracts from parasitized erythrocytes caused a dose and time dependent DNA degradation [12]. It was suggested that a progressive increase in intracellular iron levels during the further development of $P$. falciparum infected red blood cells which leads to an increased production of hydrogen peroxides $\left[\mathrm{H}_{2} \mathrm{O}_{2}\right]$ and hydroxyl $(\mathrm{OH})$ radicals that cause molecular and cellular damage [13]. Besides an enhanced intra erythrocytic oxidative stress, increased production of reactive oxygen species has also been observed outside the parasitized erythrocyte. In malaria infection, both host and parasite are under oxidative stress, increased production levels of reactive oxygen species (ROS, e.g. superoxide anion and the hydroxyl radical) are produced by activated neutrophils in the host and during degradation of haemoglobin in the parasite. The effects of reactive oxygen species in malaria can be both beneficial and pathological, depending on the amount and place of production. Enhanced reactive oxygen species production after the administration of pro-oxidants, which is directed against the intra-erythrocytic parasite, inhibits the infection both in vitro and in vivo [14,15]. However, reactive oxygen species are also involved in pathological changes in host tissue like damage of the vascular endothelial lining during a malaria infection (cerebral malaria). Pro-oxidants support the host defense against the parasite when working in or near the infected cell but potentially cause vascular damage when working on or near the vascular lining. Examples of pro-oxidants are artemisinin and its derivatives. The aim of this study therefore is to determine the gender difference on stress induced by malaria parasite infection and effect of antimalaria drug on stress index.

\section{Materials and Methods}

\subsection{Subjects Selection}

Two hundred and two confirmed malaria infected patients were recruited for the study between the ages of $15-64$ years of both sexes at the general outpatient clinic of the Federal Medical Centre, Ido-Ekiti, Ekiti State, Nigeria. One hundred and two apparently healthy malaria negative subjects were used as controls from hospital staff at Federal Medical Centre, Ido-Ekiti which serves as study control group and comprising both sexes between the ages 15 - 64 years within March 2012 and November 2013. The study was conducted with an informed consent of the patients. Ethical approval was obtained from ethical committee of Federal Medical Centre, Ido-Ekiti, Ekiti State. 


\subsection{Sample Collection}

About $7 \mathrm{ml}$ of blood sample was collected from each subject on the first day of visiting hospital as baseline sample grouped as pre anti-malaria drug treatment sample, after the patient has been clinically diagnosis for malaria infection and confirmed using malaria rapid kit, another $7 \mathrm{ml}$ of blood sample was collected on the second or third day from the same patient after taking anti-malaria drugs then grouped as post anti-malaria drug treatment sample. Out of $7 \mathrm{ml}$ blood sample collected; $3 \mathrm{ml}$ of blood sample was dispensed into plain bottles; serum was extracted to assay stress index hormone (cortisol) using ELISA method by monobind Inc. lake forest and malondialdehyde (MDA) using Thiobarbituric Acid Assay method, $1 \mathrm{ml}$ of blood sample was dispensed into fluoride oxalate bottles to assay blood glucose level using glucose oxidase test method and $3 \mathrm{ml}$ of blood sample was dispensed into lithum heparin bottle to assay lipid profile using randox kit. Malaria parasite was screened for using commercially prepared malaria rapid test kit; also thick and thin blood film was made for microscopic gold standard diagnosis of malaria parasite infection; for malaria parasite detection, malaria parasite count and malaria parasite species identification. Questionnaire was used to obtain the demographic characteristics and other relevant information for the study.

\subsection{Microscopic Diagnosis of Malaria Parasite Using Thick and Thin Stained Blood Film}

Thick blood film was made and stained using Giemsa's staining technique for malaria parasite detection and malaria parasite count. However, thin blood film was also made and stained with Leishman staining technique for plasmodium species identification as described by Monica Cheesbrough, 2005 [16]. Changes in parasitized red cells help to identity plasmodium species and to detect mixed infection of malaria parasite. The number of asexual P.falciparium and other species per 200 leukocytes were counted and if ten or more parasites were identified, then the number was recorded, a blood sample was regarded as negative if the examination of thick films failed to show the presence of asexual parasites $[17,18]$. The parasite count in relation to the leukocyte count was converted to parasite per micro litre of blood using this mathematical formula

$$
\text { parasites / ul of blood }
$$

Number of observed asexual parasites

$$
=\frac{x \text { total WBC count } / \text { ul }}{200 \text { (Number of leucocytes counted) }}
$$

\subsection{Statistical Analysis}

Data obtained were analyzed using student t-test to compare means. Analysis was performed using computer database software from the statistical package for social sciences (version 16.0 SPSS). A P-value of $<0.05$ was considered statistically significant in all clinical comparisons at $95 \%$ confidence interval.

Table 1. Mean standard deviation of gender differences on stress index, blood glucose and lipid profile in pre, post anti malaria drugs

\begin{tabular}{|c|c|c|c|c|c|c|c|c|c|}
\hline GROUPS & \multicolumn{3}{|c|}{ Pre- treatment } & \multicolumn{2}{|c|}{ Post treatment } & \multicolumn{4}{|c|}{ Control } \\
\hline Parameters & $\begin{array}{c}\text { Male } \\
(\mathrm{N}=129)\end{array}$ & $\begin{array}{l}\text { Female } \\
(\mathrm{N}=73)\end{array}$ & $\begin{array}{c}\mathrm{P} \\
\text { value }\end{array}$ & $\begin{array}{c}\text { Male } \\
(\mathrm{N}=129)\end{array}$ & $\begin{array}{l}\text { Female } \\
(\mathrm{N}=73)\end{array}$ & $\begin{array}{c}\mathrm{p} . \\
\text { value }\end{array}$ & $\begin{array}{c}\text { Male } \\
(\mathrm{N}=58)\end{array}$ & $\begin{array}{l}\text { Female } \\
(\mathrm{N}=44)\end{array}$ & $\begin{array}{c}\mathrm{p} . \\
\text { value }\end{array}$ \\
\hline CORTISOL g/dL & $23.38 \pm 3.77$ & $21.10 \pm 3.08$ & 0.08 & $18.09 \pm 7.05$ & $14.37 \pm 4.58$ & $0.01^{*}$ & $7.27 \pm 1.52$ & $5.71 \pm 1.29$ & 0.20 \\
\hline $\mathrm{MDA}$ nmol/L & $19.98 \pm 3.25$ & $19.16 \pm 2.41$ & $0.03 *$ & $16.46 \pm 6.22$ & $13.28 \pm 3.59$ & $0.00 *$ & $8.66 \pm 0.85$ & $7.86 \pm 0.68$ & $0.04 *$ \\
\hline GLUCOSE mmol/L & $5.23 \pm 0.73$ & $4.92 \pm 0.79$ & 0.08 & $4.53 \pm 0.92$ & $4.08 \pm 0.69$ & $0.05 *$ & $3.63 \pm 0.33$ & $3.57 \pm 0.31$ & 0.69 \\
\hline HDL mg/Dl & $29.58 \pm 4.66$ & $29.07 \pm 4.54$ & 0.44 & $27.54 \pm 3.48$ & $27.51 \pm 3.40$ & 0.69 & $43.07 \pm 3.86$ & $40.77 \pm 2.96$ & 0.07 \\
\hline $\mathrm{LDL} \mathrm{mg} / \mathrm{Dl}$ & $51.81 \pm 15.92$ & $55.84 \pm 16.37$ & 0.73 & $57.41 \pm 12.20$ & $60.59 \pm 12.04$ & 0.99 & $64.66 \pm 6.40$ & $59.95 \pm 6.68$ & 0.57 \\
\hline TRIG mg/dL & $77.35 \pm 19.35$ & $79.42 \pm 21.47$ & 0.18 & $86.24 \pm 17.14$ & $88.03 \pm 18.47$ & 0.17 & $99.64 \pm 12.29$ & $92.82 \pm 9.52$ & $0.04 *$ \\
\hline WBC $\times 10^{9 / L}$ & $6.82 \pm 1.81$ & $6.45 \pm 1.37$ & $0.00 *$ & $6.08 \pm 2.14$ & $5.44 \pm 1.52$ & $0.00 *$ & $4.49 \pm 0.30$ & $4.33 \pm 0.35$ & 0.24 \\
\hline MPC $\mu / L$ & $2643.00 \pm 474.85$ & $2602.20 \pm 281.73$ & $0.00 *$ & $2373.40 \pm 625.99$ & $2184.50 \pm 300.35$ & $0.00 *$ & - & $\begin{array}{c}- \\
-1.000\end{array}$ & - \\
\hline
\end{tabular}
treatment in malaria infected and control subjects

$\mathrm{P}<0.05$ significance, $\mathrm{P}>0.05$ no Significant, $\mathrm{F}$ (P-value) $=$ mean + SD of parameters compared using ANOVA.

\section{Results}

Most of the participants were from rural residence and all of them were infected with Plasmodium falciparum species; patients without malaria parasite in their blood were disqualified from the study. Out of the 202 malaria infected patients 129 (63.9\%) were males and 73 (36.1\%) were females; in control subjects 58 (56.9\%) were males and $44(43.1 \%)$ were females. The mean \pm SD of MDA in male was significantly $(\mathrm{P}<0.05)$ higher compared to female in pre, post anti malaria drug treatment and control subjects; mean \pm SD of MDA in both sexes for pre antimalaria treatment was significantly $(\mathrm{P}<0.05)$ higher compared to post anti malaria drug treatment and control subjects. Mean \pm SD of cortisol and glucose were higher in male compared to female in pre, post anti malaria drug treatment and control subjects; the difference was only significant $(\mathrm{P}<0.05)$ in post anti malaria drug treatment. However, the mean \pm SD value of cortisol and glucose were higher in both sexes for pre anti malaria drug treatment compared to post anti malaria drug treatment and control subjects. Mean \pm SD of HDL in male was higher compared to female in pre, post anti malaria drug treatment and control subjects although the difference was not statistically significant $(\mathrm{P}>0.05)$. Mean \pm SD of HDL in both sexes for post anti malaria drug treatment was lower compared to pre anti malaria drug treatment and control subjects. Mean \pm SD of LDL, triglycerides and total cholesterol in male was lower compared to female in pre, post anti malaria drugs treatment, although the difference was not statistically significant $(P>0.05)$; however, in control subjects, mean \pm SD of LDL, triglycerides and total cholesterol in male were higher compared to female, there was no statistically significant difference except in triglycerides. Mean \pm SD of LDL, triglycerides and total cholesterol in pre anti malaria drug treatment was lower in both sex compared to post anti malaria drug treatment and control subjects. Mean \pm SD of WBC in male was higher compared to female in pre, post anti malaria drug treatment and control subjects. The difference was significant $(\mathrm{P}<0.05)$ except in control 
subjects. Mean \pm SD of WBC in both sexes for pre anti malaria drug treatment was higher compared to post anti malaria drug treatment and control subjects. Mean \pm SD of MPC in male was significantly $(\mathrm{P}<0.05)$ higher compared to female in pre and post anti- malaria drug treatment. Mean \pm SD of MPC for pre anti-malaria drug in both sex was significantly $(\mathrm{P}<0.05)$ higher compared to post anti-malaria drug treatment. Since control subject were at infected with malaria, hence MPC was null as shown in the Table 1.

\section{Discussion}

Malaria parasite counts is the most important aspects of reporting malaria infections, it estimate the level of parasitemia present in the blood. In this present study, the mean value of malaria parasite count and stress index in male was significantly higher compared to female in both pre-treatment and post anti-malaria drug treatment; this findings was supported by [19] stated that the prevalence of Plasmodium infection was higher in male than in female malaria infected patients; concluded that the cause could be due to the fact that males expose their bodies more than females when the weather is hot and thus increases their chances of being bitten by the mosquito. Females, on the other hand, are usually not naked and tend to stay indoors, helping out with household chores. This reduces their contact with the mosquito vector. Also, studies have shown that females have better immunity to parasitic diseases and this was attributed to genetic and hormonal factors [20]. However, there is some information on oxidative stress in men showing a prooxidant effect of testosterone [21]. In female decreased superoxide anion production by the endothelium in response to estrogen is considered to contribute to vascular protective properties of estrogen [22]. However, the level of parasitemia in post anti-malaria drug treatment was lower compared to pre anti-malaria drug treatment in both gender; this was due to effect of anti-malaria drug used during treatment $[23,24]$ supported that, patients with high parasite count have more severe and complicated course, also stated that the level of parasitaemia is useful as one of the criteria in defining "severe $P$. falciparum malaria” and to monitor the effect of anti-malarial therapy. In this present study, the level of stress induced by malaria parasite ( $p$. falciparum) in male was higher compared to female which reflect with high level of cortisol, MDA and glucose in male both in pre-treatment and post antimalaria drug treatment. Cortisol, MDA and glucose level in control subjects were observed lower compared to value obtained in pre-treatment and post-treatment. Supporting this study, high cortisol is expected before malaria treatment due to the maintained stress induced by malaria parasite but on treatment a decline in the levels of cortisol is found in maintained stress [25]. Cortisol, MDA and glucose levels would probably return to normal. This can be explained by the fact that fever reflects a time of blood cell lysis, releasing parasite antigens into the circulation which in turn, induce the release of cytokines. These cytokines stimulate the thermo-regulatory centre in the hypothalamus as well as the HPA axis, and this fact explain the higher cortisol levels during pre-treatment of malaria infection with schizogony therefore representing an acute stimulus of the HPA axis. Cortisol level tended to decline during the course of the disease and treatment, a finding that might be explained by the fact that the patient will acquire a certain resistance to malaria over time [26]. Tim et al., 1998 [27] reported that there was significant rise in serum cortisol level of malaria patients compared to control. These results demonstrate the presence of increased cortisol levels in patients with $P$. falciparum malaria infection, indicating stimulation of the HPA axis in these patients. Cortisol levels will be declining as the clinical condition improves and parasitemia decreases, probably due to a lower cytokine production. Acute stimulation of the HPA axis by stress induced in malaria infection leads to the release of cortisol and other steroids produced and secreted by the adrenal glands resulted in increased cortisol concentrations throughout the stimulus, a reduction in the stimulation of the HPA axis occurs with clinical improvement [28]. Tim et al., 1998 [27] demonstrates higher serum cortisol level in acute stage of malaria infection than corresponding convalescent levels. Inamo et al., 1991 [29] reported a two - fold increase in cortisol levels during febrile of malaria infection. The glucose metabolism during malaria infection is affected by several factors, including drug treatment, fever, parasite metabolism, hormonal changes, cytokines, fasting and gastrointestinal disturbances [30]. Hypoglycaemia occurs during the management of patients with malaria, it is well known that anti-malaria drug stimulates diabetes relevant parameters, such as increased plasma insulin concentrations and hypoglycaemia this fact supported the findings in this present study with low glucose level in post anti-malaria drug treatment compared to pre antimalaria drug treatment. Davis et al., 1993 [31] reported that in adults with severe malaria infection, glucose production was increased by $50 \%$ compared to convalescence. Increased glucose production in malaria infection especially in severe or in cerebral malaria was entirely dependent on gluconeogenesis, in non-severe malaria, glucose production is increased by approximately $25 \%$, and gluconeogenesis contributes approximately $87 \%$ to total glucose production [33]. Cytokines, counterregulatory hormones and precursor supply are all potentially implicated in the regulation of glucose production in malaria infection. High plasma cortisol may stimulate gluconeogenesis in humans [33] increase in plasma glucose concentration supports the idea of a major role for cortisol in the stimulation of gluconeogenesis in patients with malaria. However, increase in plasma cortisol associated with a major stimulation of gluconeogenesis in the patients with non-severe malaria [32]. The plasma glucose concentration is the result of glucose production and glucose uptake. Low serum lipid level observed in male compared to female in pre and post anti-malaria drug treatment in this study, was not statistically significant but reflect degree of stress induced by malaria infection in both gender and effect of antimalaria drugs during treatment. This is supported with the fact that; rapidly growing malaria parasite requires large amounts of lipids for increase in surface area and volume of its internal membranes and certain serum lipid fractions may favour the onset and/or severity of malaria infection. Contrarily, Kittl et al., 1992 [34] have shown no correlation between severity of malaria attacks and extent of HDL - cholesterol decrease. Human serum HDL is 
necessary for $P$. falciparum in in-vitro culture. Lipids have been observed to play important roles in pathological changes observed in malaria infection and are implicated in the production of immunity against diseases [35].

\section{Conclusion}

Plasmodium infected human erythrocytes are under increased oxidative stress exerted by the malaria parasite capable of generating reactive oxygen species within the erythrocytes. Stress induced by malaria parasite was observed higher in male compared to female; gender norms and values that influence the division of labour, leisure patterns, and sleeping arrangements can influence different patterns of exposure to mosquitoes for men and women which responsible for differences in stress induced by malaria parasite among the gender; during malaria treatment, the level of stress induced by malaria parasite was decline due to the effect of anti-malaria drug used.

\section{References}

[1] UNICEF (2000). Rolling back malaria goals. A United Nation Children's Fund document. 2000; 16pp.

[2] Alaribe AAA, Ejekie GC, Ezedinachi ENU. The ecology of Bain BJ (1996). Ethnic and sex differences in the total and differential white cell count and platelet count. J Clin Pathol. (2006) 49: 664666.

[3] Rahman S. Gender aspects and women's participation in the control and management of malaria in central Sudan. Social Science and Medicine, (1995) 42(10).

[4] World Health Organization (WHO, 2010). Basic Malaria Microscopy. Part 1. Learner's Guide 2010. 2nd ed. Geneva.

[5] Das, B.S., J.K. Patnaik, S. Mohanty, S.K. Mishra, D. Mohanty, S.K. Satpathy and T.K. Bose. Plasma antioxidants and lipid peroxidation products in falciparum malaria. Am. J. Trop. Med. Hyg., (1993) 49: 720-725.

[6] Sibmooh N, Pipitaporn B, Wilairatana P, Dangdoungjai J, Udomsangpetch R, Looareesuwan S, Chantharaksri U: Effect of artemisinin on lipid peroxidation and fluidity of the erythrocyte membrane in malaria. Biol Pharm Bull (2000), 23: 1275-1280.

[7] Atamma H, Ginsburg H: Origin of reactive oxygen species in erythrocytes infected with Plasmodium falciparum. Mol Biochem Parasitol, (1993) 61: 231-242.

[8] Haynes RK. Artemisinin and derivatives: The future for malaria treatment? Curr Opin Infect Dis (2001) 14: 719-726.

[9] Jayshree R S, Ganguly N K, Dubey M L, Mohan K, Mahajan R C. Generation of reactive oxygen Species by blood monocytes during acute P. Knowiesis infection in rhesus monkeys APMIS (1993) 101: 762-766.

[10] Halliwell B. Drug anti oxidant effects; A basis for drug selection? (1991) Drugs 42(4): 569-605.

[11] Rice - Evans C, Bruckdorfr K R. Free radical, lipoproteins and cardiovascular dysfunction. Molee Aspects Med (1992) 13: 1-111.

[12] Golenser, J., E. Marva and M. Chevion,. The survival of plasmodium under oxidant stress. Parasitol. Today, (1991) 7: 142146.

[13] Vander jagt DL, Hunsaker LA, Campos N.M, Scaletti J.V. Localization and characterization of haemoglobin degrading aspartic proteinases from the malaria parasite plasmodium falciparum. Biochem Biophys Acta (1992) 1122: 256-264.

[14] Kulkarni, A.G., A.N. Suryakar, A.S. Sardeshmukh and D.B. Rathi. Studies on Biochemical changes with special reference to oxidant and antioxidant in malaria patients. Indian J. Clin. Biochem. (2003), 18: 136-149.

[15] Becker K, Tilley L, Vennerstron JL, Roberts D, Rogerson S, Ginsburg H. oxidative stress in malaria parasite infected erythrocytes: Host-Parasite Interactions Int J Parasitol; (2004) 34(2): 163-189.

[16] Monica Cheesbrough (2005). Discrete Laboratory Practice in Tropical Countries Part 1, Cambridge Second Editions. Published by Press Syndicate of the University of Cambridge, chp. 5, page 247-258.

[17] Dayachi F, Kabongo L, Ngoie K. Decreased mortality from Malaria in children with symptomatic HIV infection. Int. Cont. AIDS (1991) 2: 164.

[18] Warhurst, D.C. and J.E. Williams,. Acp Broadsheet no 148. July 1996. Laboratory diagnosis of malaria. J. Clin. Pathol., (1996) 49 : 533-538.

[19] Akanbi, O.M., J.A. Badaki, O.Y. Adeniran and O.O. Olotu,. Effect of blood group and demographic characteristics on malaria infection, oxidative stress and haemoglobin levels in South Western Nigeria. Afr. J. Microbiol. Res., (2010) 4: 877-880.

[20] Zuk M, McKean KA. Sex differences in parasite infections: patterns and processes. Inter J Parasitol; (1996) 26: 1009-23.

[21] Dincer, Y., E. Ozen, P. Kadioglu, H. Hatemi and T. Akcay. Effect of sex hormones On lipid peroxidation in women with polycystic ovary syndrome, healthy women and men. Endocrine Res., (2001) 27: 309-316.

[22] Barbacanne, M.A., J. Rami, J.B. Michel, J.P. Souchard and M. Philippe. Estradiol increases rat aorta endothelium-derived relaxing factor (EDRF) activity without changes in endothelial NO synthase gene expression: Possible role of decreased endotheliumderived superoxide anion production. Cardiovascular Res., (1999) 41: 672-681.

[23] McElroy PD, Beier JC, Oster CN, Beadle C, Sherwood JA, Oloo AJ. Predicting outcome in malaria: correlation between rate of exposure to infected mosquitoes and level of Plasmodium falciparum parasitemia. Am J Trop Med Hyg; (1994) 51: 523-32.

[24] Murthy GL, Sahay RK, Srinivasan VR, Upadhaya AC, Shantaram V, Gayatri K. Clinical profile of $P$. falciparum malaria in a tertiary care hospital. J Indian Med Assoc; (2000) 98: 160-2, 169.

[25] Ringwald, P., Peyron, F.,Vuillez, J.P., Touze, J.E., Le Bras, J., Deloron, P. Levels of cytokines in plasma during Plasmodium falciparum malaria attacks. J. Clin. Microbiol. (1991) 29, 20762078.

[26] Rosana Maria Feio Libonati, Berenice Bilharinho de Mendonc, Jos'e Antºnio Mau'es, Juarez Antonio Simoes Quaresma, Jos'e Maria de Souza. Some aspects of the behavior of the hypothalamus-pituitary-adrenal axis in patients with uncomplicated Plasmodium falciparum malaria: Cortisol and dehydroepiandrosterone levels Acta Tropica (2006) 98: 270-276.

[27] Tim Shwe, Myo Khin, Hia Mim, Koko Hia, Yin Yin Win, Kyin Htwe, and Thein Myint Thu. Serum cortisol levels in patients with uncomplicated and cerebral malaria (1998) vol. 29 No 1: 1-4.

[28] Parker, L.N., Levin, E.R., Lifrak, E.T. Evidence for adrenocortical adaptation to severe illness. J. Clin. Endrocrinol. Metab. (1985) 60, 947-952.

[29] Inamo $\mathrm{Y}$, Iakeuchi $\mathrm{S}$, Okuni $\mathrm{M}$. Host responses and neuroendocrinological changes in pyrexia in childhood. Acta pacdiat jpn (overseas ed) (1991) 33: 628-632.

[30] Davis TM, Binh TQ, Thu le TA, Long TT, Johnston W, Robertson $\mathrm{K}$, Barrett PH: Glucose and lactate turnover in adults with falciparum malaria: effect of complications and antimalarial therapy. Trans R Soc Trop Med Hyg, (2002) 96:411-417.

[31] Davis TM, Looareesuwan S, Pukrittayakamee S, Levy JC, Nagachinta B, White NJ. Glucose turnover in severe falciparum malaria. Metabolism; (1993) 42:334-340.

[32] Dekker E, Romijn JA, Ekberg K, Wahren J, Van Thien H, Ackermans MT, Glucose production and gluconeogenesis in adults with uncomplicated falciparum malaria. Am J Physiol; (1997) 272:E1059-1064.

[33] Tayek JA, Katz J. Glucose production, recycling, Cori cycle, and gluconeogenesis in humans: relationship to serum cortisol. Am J Physiol; (1997) 272:E476-484.

[34] Kittl EM, Diridl G, Lenhart V, Neuwald C, Tomasits J, Pichler H, Bauer k: HDL-cholesterol as a sensitive diagnostic criterion in malaria. Wien Klin Wochenschr, (1992) 104:21-24.

[35] Beckwith. R, Schenkel R. H. and Silverman P. H. Qualitative analysis of phospholipids isolated from nonviable Plasmodium antigen, Experimental Parasitology, (1975) vol. 37, no. 2, pp. 164172. 